\title{
Renormalizing Entanglement Distillation
}

\author{
Stephan Waeldchen, ${ }^{1}$ Janina Gertis, ${ }^{1}$ Earl T. Campbell, ${ }^{2}$ and Jens Eisert ${ }^{1}$ \\ ${ }^{1}$ Dahlem Center for Complex Quantum Systems, Freie Universität Berlin, 14195 Berlin, Germany \\ ${ }^{2}$ Department of Physics and Astronomy, University of Sheffield, Sheffield S3 7RH, United Kingdom
}

(Received 2 May 2015; published 15 January 2016)

\begin{abstract}
Entanglement distillation refers to the task of transforming a collection of weakly entangled pairs into fewer highly entangled ones. It is a core ingredient in quantum repeater protocols, which are needed to transmit entanglement over arbitrary distances in order to realize quantum key distribution schemes. Usually, it is assumed that the initial entangled pairs are identically and independently distributed and are uncorrelated with each other, an assumption that might not be reasonable at all in any entanglement generation process involving memory channels. Here, we introduce a framework that captures entanglement distillation in the presence of natural correlations arising from memory channels. Conceptually, we bring together ideas from condensed-matter physics - ideas from renormalization and matrix-product states and operators - with those of local entanglement manipulation, Markov chain mixing, and quantum error correction. We identify meaningful parameter regions for which we prove convergence to maximally entangled states, arising as the fixed points of a matrix-product operator renormalization flow.
\end{abstract}

DOI: 10.1103/PhysRevLett.116.020502

It has long been noted in the field of quantum information science that entanglement constitutes the key resource in various information processing and specifically communication tasks [1]. Secure quantum key distribution necessarily relies on entanglement, even in prepare-and-measure schemes [2-4]. A central goal in quantum information science has been the development of techniques to transform less useful forms of entanglement into more suitable ones, and to enhance our understanding of the laws governing the manipulation of entanglement. The task of entanglement distillation specifically captures the resource character of entanglement, in that it aims at preparing maximally entangled states from noisy or less-entangled ones [1]. The concept of distillable entanglement grasps the maximum rate at which this is asymptotically possible, starting from a collection of many identically prepared quantum systems; hence, this is of profound interest in the conceptual foundations of the field. Distillation steps are part of quantum repeater protocols [5-7], necessary to distribute entanglement over arbitrary distances using noisy quantum channels: In such a scheme, entanglement is established between different repeater stations and transferred to the final designated nodes via suitable entanglement-swapping steps. Distillation schemes thought of in this context are often iterative schemes, such as the recurrence protocol $[1,8]$ and deterministic protocols based on error-correction codes [1,9-11]. While iterative schemes do not achieve the maximum rates set by the distillable entanglement, they require less sophisticated and more practically feasible operations. The silent assumption in almost all of the proposed distillation schemes, however, is that the initial resources have been identically prepared and show no correlations whatsoever. While this is surely a good assumption in many preparations, it might not be reasonable in others. Whenever memory effects or channels [12-15] are involved, one expects some correlations between the involved entangled pairs, going beyond an independent identically distributed (IID) setting. These correlations are expected to decay rapidly over several pairs sent through a channel, reflecting the natural correlation structure arising from a memory channel (see Fig. 1). The mathematical definition of distillable entanglement in the presence of correlations has been developed [16,17] in the context of quantum information theory beyond the IID assumption [15,18-20]. In fact, even under correlations, a positive asymptotic rate of entanglement distillation can be achieved using the hashing protocol, but such methods require coherent state manipulation over all specimens of the correlated quantum systems and do not make use of the short-range structure of realistic correlations. Yet, the important practical problem of actually iteratively distilling entanglement from correlated pairs arising from quantum memory channels is still wide open.

(a)

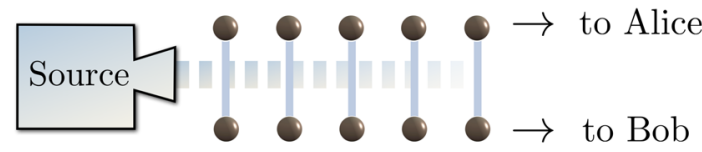

(b)

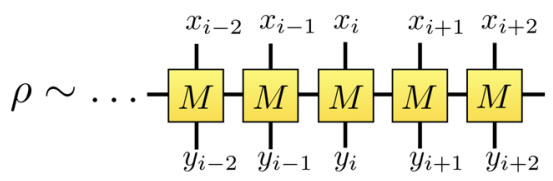

FIG. 1. (a) Source with memory emitting weakly correlated photon pairs and (b) the MPO $\rho$ naturally describing this setting. 
In this Letter, we propose a conceptually novel way forward to solve this problem, bringing together ideas from entanglement theory with those of condensed-matter theory, specifically of renormalization techniques and tensor network methods. We identify the natural class of states arising from preparations and memory channels as bipartite matrix-product operators (MPOs) $[21,22]$. These are usually considered in the condensed-matter context to capture thermal many-body states or those arising from open systems dynamics [21-24]. Entanglement distillation is then identified as a renormalization of bipartite matrixproduct operators. The methods are inspired by and derive from renormalization [25] of matrix-product states [26-31], again from many-body theory.

We show that both the recurrence protocol and an errorcorrection-based protocol [1] converge to pairs of maximally entangled pure states for suitably correlated pairs that are naturally described by a MPO.

Setting and formalism.-We consider a sequence of $L$ pairs of qubits, where two parties (say Alice and Bob) each hold one qubit from each pair. The focus on qubits is set for simplicity of notation only; it is clear that the same framework can be applied to systems of other physical dimension. These pairs are entangled, as well as correlated with each other, as a consequence of the preparation procedure involving stationary quantum memory effects. A natural preparation exhibiting such a memory involves an auxiliary quantum system $C$ of some dimension $d$ that embodies all the degrees of freedom of the memory. The state is then prepared in a sequential fashion, with the memory unitarily interacting with the first entangled pair, then the second, and so on [13,27,32]. A state generated in this way is given by a matrix-product state, if it is pure, or a matrix-product operator in case of noisy mixed states $[21,22]$, as they are considered here, with $d$ taking the role of the bond dimension. The decay of memory effects in the distance between the entangled pairs naturally emerges in this construction. We introduce here how a naturally correlated bipartite MPO arises from this setting. More specifically, we work in a numerically indexed Bell basis $\left(\left|\phi_{1}\right\rangle,\left|\phi_{2}\right\rangle,\left|\phi_{3}\right\rangle,\left|\phi_{4}\right\rangle\right)$, more commonly labeled as $\left(\left|\phi^{+}\right\rangle,\left|\phi^{-}\right\rangle,\left|\psi^{+}\right\rangle,\left|\psi^{-}\right\rangle\right)$. We consider a sequence of $L$ pairs of qubits, with basis vectors $\left|\Phi_{\mathbf{x}}\right\rangle=\left|\phi_{x_{1}}\right\rangle\left|\phi_{x_{2}}\right\rangle \ldots\left|\phi_{x_{L}}\right\rangle$, where Alice holds the first qubit of each pair and Bob holds its partner. Translationally invariant mixed states reflecting stationarity of the source are described in the MPO language as

$$
\left\langle\Phi_{\mathbf{x}}|\rho| \Phi_{\mathbf{y}}\right\rangle=\operatorname{Tr}\left[M^{x_{1}, y_{1}} M^{x_{2}, y_{2}} \ldots M^{x_{L}, y_{L}}\right] .
$$

Purely for simplicity of notation, we take periodic boundary conditions here. The dimension of the matrices $M^{x, y} \in \mathbb{C}^{d \times d}, \quad x, y \in\{1, \ldots, 4\}$ limits the correlations between pairs, and by increasing this bond dimension $d$, arbitrary quantum states can be described in this formalism.
There is a gauge freedom in our choice of MPO matrices; for any invertible $S$, mapping $M^{x, y} \mapsto S M^{x, y} S^{-1}$ will give an alternative description of the same physical state. Generally, 16 matrices are needed for the description of each pair, reflecting the two-particle density matrix. However, without loss of generality we take $\rho$ to be a Bell diagonal state, which can be achieved using a suitable local group twirl over the Pauli group [1]. For this reason we use the shorthand $A=M^{1,1}, B=M^{2,2}, C=M^{3,3}$, and $D=M^{4,4}$. Without loss of generality, we consider the distillation of maximally entangled $\phi^{+}$pairs. The " $A$ " matrix will be the dominant matrix; we will call the others noise matrices.

Protocols and renormalization.-An $N \rightarrow M$ iterative protocol for entanglement distillation of IID states will act on $N$ pairs at a time and output $M$ pairs. Given $k N$ pairs, we perform $k$ implementations in parallel. In the MPO setting, pairs are not IID and we must specify which pairs are distilled together. We choose neighboring pairs so the first $N$ pairs are distilled, while simultaneously the next $N$ pairs are distilled, and so on. This natural choice has the practical merit of respecting locality, and has the additional advantage that the output state is easily shown to again be a MPO of the same bond dimension (see Fig. 2). Every iteration of the distillation protocol now acts as a map from a MPO on one scale to the subsequent one and reduces the chain length from $L$ to $L M / N$. After each step, a positive MPO is retained [33]. Indeed, it can be naturally seen as process of MPO renormalization, this being a mixed-state and bipartite analogue of the renormalization of matrix product states discussed in Ref. [25]. After the $n$th step, we label the MPO operators $\left\{A_{n}, B_{n}, C_{n}, D_{n}\right\}$, where the initial raw state provides the $n=0$ matrices. We prove several results on convergence to entangled states that show the functioning of the schemes; proofs that can also be interpreted as convergence proofs for renormalization flow of the MPOs. Intuitively, one can say that in many practically relevant settings, the entanglement and correlations between pairs are being "renormalized" into more useful entanglement shared between Alice and Bob, to be employed in subsequent key generation.

Recurrence protocol.-The recurrence protocol is a $2 \rightarrow 1$ iterative protocol that uses postselection and, hence, (a)

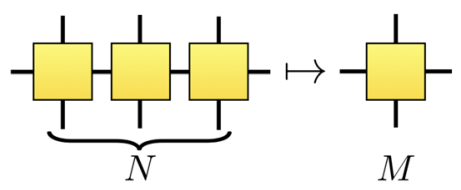

(b)

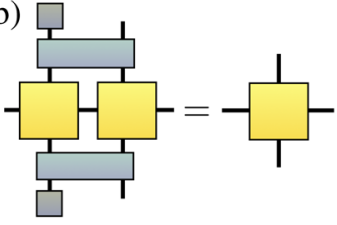

FIG. 2. Renormalization schemes of a MPO. (a) $N$ pairs are mapped to $M$ in an $N \rightarrow M$ scheme. (b) In the recurrence protocol, two neighboring sites are distilled. Contraction of the tensor network leads to the MPO at the subsequent scale. 
is probabilistic. Each step is a slightly improved version [8] of the recurrence scheme [34]. In the uncorrelated case, every pair can be postselected individually, if memories are available, thus keeping all successfully measured pairs. In the MPO setting, to retain translational invariance over these $L / 2$ sites, we modify recurrence to globally postselect, requiring correct measurement outcomes for all pairs. If the recurrence protocol fails at one location, we discard the chain rather than replacing the failures with new pairs. Applied to a length $L$ chain, it becomes an $L \rightarrow L / 2$ iterative protocol. Though global postselection reduces the overall success probability, it does benefit from avoiding long storage times in quantum memories, which are otherwise incurred when waiting for communication of measurement results. Cast into the MPO language, the iteration formula after two steps is

$$
\begin{gathered}
A_{n+2}=\left(A_{n}^{2}+B_{n}^{2}\right)^{2}+\left(C_{n}^{2}+D_{n}^{2}\right)^{2}, \\
B_{n+2}=\left\{A_{n}, B_{n}\right\}^{2}+\left\{C_{n}, D_{n}\right\}^{2}, \\
C_{n+2}=\left\{A_{n}^{2}+B_{n}^{2}, C_{n}^{2}+D_{n}^{2}\right\}, \\
D_{n+2}=\left\{\left(A_{n}, B_{n}\right),\left(C_{n}, D_{n}\right)\right\},
\end{gathered}
$$

where curly brackets denote the anticommutator. After two steps, the matrices are being regauged and rescaled. Replacing matrices by commuting scalars recovers the original IID result. Proving convergence of the initial MPO in the state $\rho$ to the maximally entangled state $\phi^{+}$is achieved by showing that the noise matrices vanish exponentially faster than the $A_{n}$ matrices. Specifically, an appropriate ratio of norms will be exponentially suppressed. We define a norm $\|M\|$ in terms of a channel $\mathcal{M}$ isomorphic [21] to $M$, so that $\|M\|=\|\mathcal{M}\|_{1 \rightarrow 1}$, where we use the induced "1-to-1" norm [35]. We introduce the noise contribution of the coefficient matrices $B_{n}, C_{n}$, and $D_{n}$ as

$$
\epsilon_{n}=\max \left(\left\|\mathcal{B}_{n}\right\|_{1 \rightarrow 1},\left\|\mathcal{C}_{n}\right\|_{1 \rightarrow 1},\left\|\mathcal{D}_{n}\right\|_{1 \rightarrow 1}\right) .
$$

Because of norm submultiplicativity, one finds that $\epsilon_{n}$ vanishes with increasing $n$ provided that the initial value $\epsilon_{0}$ is sufficiently small. However, ensuring $A_{n+2}$ stays large is difficult. To do so, we shall adjust the MPO gauge after two steps, regauging this using a suitable gauge transformation and rescaling, so that $\mathcal{A}_{n+2}$ is trace preserving and, hence, $\left\|\mathcal{A}_{n+2}\right\|_{1 \rightarrow 1}=1$. To quantify how much the gauge transformation changes the matrix norm, we rely on the ergodicity coefficient of the matrices,

$$
\tau(\mathcal{M})=\max _{\operatorname{Tr}[\sigma]=0} \frac{\|\mathcal{M}(\sigma)\|_{1}}{\|\sigma\|_{1}},
$$

which allows a quantification of how rapidly a channel mixes input states into the channel's stationary state. We are interested in the ergodicity of $\mathcal{A}_{n}$, for which we use the shorthand $\tau_{n}:=\tau\left(\mathcal{A}_{n}\right)$. We are now ready to state the first main result, which provides sufficient conditions for entanglement distillation using the recurrence protocol.

Theorem 1 [Convergence in the recurrence protocol] Given a translationally invariant Bell-diagonal MPO with coefficient matrices $A_{0}, B_{0}, C_{0}$, and $D_{0}$, the iterative application of the recurrence protocol leads to convergence to uncorrelated pairs in the maximally entangled state $\phi_{+}$ for $\epsilon_{0}<\left(1-\tau_{0}^{4}\right) / 7\left(1+\tau_{0}^{4}\right)$.

The convergence is illustrated in Fig. 3. The proof is presented in full length in the Supplemental Material [36], but we will sketch it here.

To prove convergence, we need to show that the noise matrices go down exponentially fast, while $A_{n}$ stays large. The first part can be shown by taking into account a double step of the protocol after which all norms of the noise matrices are at least of order $\epsilon_{n}^{2}$. However, to ensure the contribution of the dominant matrix stays large, $\mathcal{A}_{n+2}$ has to be regauged such that it stays trace preserving. With $\mathcal{A}_{n}^{\dagger}\left(\xi_{n}\right)=\lambda_{n} \xi_{n}$ (where $\lambda_{n}$ is the largest such eigenvalue), the gauge transformation $S_{n}=\sqrt{\xi_{n}} \otimes \sqrt{\xi_{n}}$ and the rescaling by $\lambda_{n}^{-1}$ will recover trace preservation, provided $\xi_{n}$ is invertible. Using submultiplicativity, we find that

$$
\epsilon_{n+2} \mapsto \lambda_{n+2}^{-1} \kappa(\mathcal{S})_{n+2} \epsilon_{n+2},
$$

where $\kappa\left(\mathcal{S}_{n}\right)=\left\|\mathcal{S}_{n}\right\|_{1 \rightarrow 1}\left\|\mathcal{S}_{n}^{-1}\right\|_{1 \rightarrow 1}$ is the condition number of $\mathcal{S}_{n}$. We further show that

$$
\kappa\left(\mathcal{S}_{n}\right) \leq \frac{1}{1-2 k_{n}}, \quad k_{n}=\frac{1+\tau_{n}^{4}}{1-\tau_{n}^{4}}\left(4 \epsilon_{n}^{2}+10 \epsilon_{n}^{4}\right) .
$$

The proof bears similarities to the perturbation of the steady state of a trace-preserving quantum Markov chain [38]. If $\mathcal{A}_{n}$ is a rapidly mixing channel with small $\tau_{n}$, then $\mathcal{A}_{n}^{4}$ is also rapidly mixing. Before the gauge transformation, $A_{n+2}$ is a sum of $A_{n}^{4}$ and some small noise matrices. The more rapidly mixing a channel (small $\tau_{n}$ ), the more its eigenstates are robust against the perturbative addition of noise matrices (small $\epsilon_{n}$ ), and the dual eigenstate $\xi_{n+2}$ stays

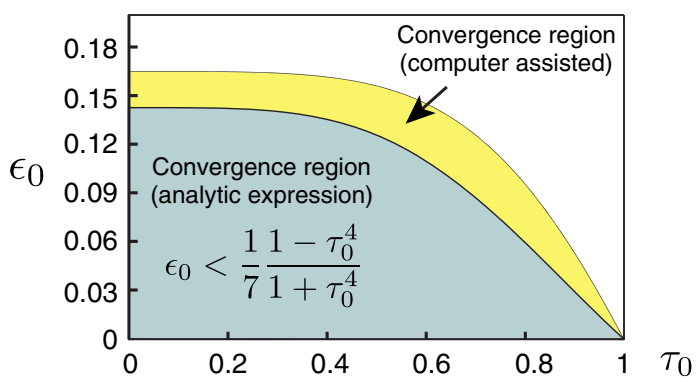

FIG. 3. Region of convergence for the recurrence protocol. The area under the blue line is the region fulfilling the conditions given in Theorem 1. The yellow region is a slightly improved bound that can be obtained with computer assistance, but for which we have no closed-form expression. 
close to $\mathbb{1}$, as we show rigorously in a spirit similar to the ideas of Ref. [39].

Although ergodicity is not a matrix norm, it has similar properties such as subadditivity and submultiplicativity, from which one can derive an upper bound on $\tau_{n+2}$ in terms of $\tau_{n}$ and $\epsilon_{n}$ so that $\tau_{n+2} \leq \tau_{n}^{4}+f\left(\epsilon_{n}, \tau_{n}\right)$ where $f$ is a slight correction that vanishes as $\epsilon_{n} \rightarrow 0$. This occurs because a double step of the protocol raises the ergodicity coefficient of $A$ to the fourth power, but regauging the MPO results in the adjustment $f$. We derive bounds on the pair $\left(\epsilon_{n+2}, \tau_{n+2}\right)$ in terms of $\left(\epsilon_{n}, \tau_{n}\right)$. From these, we iteratively determine the region of convergence to the fixed point $(0,0)$ with computer assistance, shown in Fig. 3. Also shown in this figure is an analytic bound for which we show $\left(\epsilon_{n}, \tau_{n}\right) \rightarrow(0,0)$ without computer assistance. Finally, convergence in MPO operators again entails convergence of the density matrix.

Five-qubit protocol.-For every error-correcting code that encodes $k$ qubits into $N$ physical qubits, there exists an iterative $N \rightarrow k$ one-way entanglement distillation protocol [1]. In these protocols, noise information is extracted by local measurements, but instead of postselecting when errors are detected we attempt to correct them by determining the smallest weight error consistent with the measurement data. The advantage over the recurrence protocol is that this protocol is deterministic, and that one-way distillation schemes require much less classical communication. Further, this means that deterministic protocols may be implemented without modification, such as the global postselection we introduced for recurrence. In particular, we consider one-way entanglement distillation using the 5 -qubit code (so, a $5 \rightarrow 1$ protocol), which is the smallest known code capable of correcting any single qubit error. We again find a closed form for the map acting on the coefficient matrices in each iteration, though we omit it here as each expression contains $4^{4}$ terms. We further introduce the transfer matrix $E_{n}=A_{n}+B_{n}+C_{n}+D_{n}$ with corresponding channel $\mathcal{E}_{n}$.

Theorem 2 [Error correction] Given a translationally invariant Bell-diagonal MPO with coefficient matrices $A_{0}$, $B_{0}, C_{0}$, and $D_{0}$, the iterative application of the 5-qubit error-correcting code leads to convergence to uncorrelated pairs in the maximally entangled state $\phi_{+}$for $\epsilon_{0} \leq 1 / 33$. $\epsilon_{0}$ is defined as above in the gauge where $\mathcal{E}_{0}$ is trace preserving.

Again, the full proof is provided in the Supplemental Material [36]. The line of the argument is similar to the postselective case; we show that with a growing number of iterations $n$, the contribution of the dominant matrix $A_{n}$ grows exponentially faster than the contribution of the noise matrices, but in the deterministic case, we can use our gauge freedom to make $\mathcal{E}_{n}$ trace preserving. Since we do not postselect, we do not need to renormalize in every round. The transfer matrix is always mapped to its fifth power, $E_{n+1}=E_{n}^{5}$, and the trace-preserving property is conserved without regauging. Thus, if we initially choose a gauge where the corresponding map $\mathcal{E}_{0}$ is trace preserving, the transfer matrix will keep this property over the course of the iteration. Using combinatorial arguments, we then prove that for suitably small $\epsilon_{0}, \epsilon_{n}$ converges to zero, entailing convergence in fidelity of the physical state.

Numerical studies and physical Hamiltonians.-To be rigorous, our proof made several pessimistic assumptions, and so many MPOs may converge despite not satisfying the conditions of our theorems. As such, we have also performed numerical studies on randomly drawn matrices. Numerical analysis of randomly drawn MPO operators indicate that strong correlations can also be beneficial and can enable distillation at noise levels well above the rigorous threshold, even above the level tolerated by IID states. We also considered the generation of MPO states where qubits sequentially interact with a common bath through a Heisenberg interaction. Benchmarking against IID pairs, we find these physical memory channels fall into both beneficial and pernicious classes depending on the exact parameter regime (full details in Supplemental Material [36]).

Perspectives. - In this Letter, we have introduced a framework of renormalizing entanglement in order to achieve iterative entanglement distillation in the presence of natural correlations. On intuitive grounds, one might expect that if the MPO is only weakly correlated between the pairs and the reduced density matrix of a single pair is sufficiently close to a Bell pair, the distillation protocols should behave similarly to the IID case. Here, we have proven that, indeed, iterative protocols known to work for IID pairs also give rise to feasible entanglement distillation, with no modification of the protocol needed. Specifically, we have identified threshold fidelities and conditions on the correlation between the pairs to ensure the convergence of correlated pairs described by a MPO to a number of independent maximally entangled pure states. Sometimes, correlations are even found to help significantly.

The program initiated here shows that correlations are not necessarily a disadvantage, and one does not have to aim at de-correlating pairs or resetting preparation procedures, which are steps that will take time and will in practice lead to further entanglement deterioration. Conceptually speaking, we have added a new mindset to the theory of beyond IID quantum information processing [17], shifting the emphasis away from protocols involving fully general asymptotic state manipulation. This work shows that such correlations can be largely renormalized away, again with no modification to the schemes applied. We hope that this Letter triggers further studies on entanglement distillation and repeater protocols in the presence of realistic memory effects, as well as of renormalizing matrix-product operators.

We acknowledge funding from the BMBF (Q.com), the DFG (SPP), the European Union (SIQS, RAQUEL, AQuS), the EPSRC (Grant No. EP/M024261/1), and the ERC (TAQ). We also acknowledge comments from Martin Kliesch. 
[1] C. H. Bennett, D. P. DiVincenzo, J. A. Smolin, and W. K. Wootters, Mixed-state entanglement and quantum error correction, Phys. Rev. A 54, 3824 (1996).

[2] N. Gisin, G. Ribordy, W. Tittel, and H. Zbinden, Quantum cryptography, Rev. Mod. Phys. 74, 145 (2002).

[3] N. Gisin and R. Thew, Quantum communication, Nat. Photonics 1, 165 (2007).

[4] M. Curty, M. Lewenstein, and N. Lütkenhaus, Entanglement as a Precondition for Secure Quantum Key Distribution, Phys. Rev. Lett. 92, 217903 (2004).

[5] H. J. Briegel, W. Dür, J. I. Cirac, and P. Zoller, Quantum Repeaters: The Role of Imperfect Local Operations in Quantum Communication, Phys. Rev. Lett. 81, 5932 (1998).

[6] L. M. Duan, M. D. Lukin, J. I. Cirac, and P. Zoller, Longdistance quantum communication with atomic ensembles and linear optics, Nature (London) 414, 413 (2001).

[7] L. Childress, J. M. Taylor, A. S. Sørensen, and M. D. Lukin, Fault-Tolerant Quantum Communication Based on SolidState Photon Emitters, Phys. Rev. Lett. 96, 070504 (2006).

[8] D. Deutsch, A. Ekert, R. Jozsa, C. Macchiavello, S. Popescu, and A. Sanpera, Quantum Privacy Amplification and the Security of Quantum Cryptography Over Noisy Channels, Phys. Rev. Lett. 77, 2818 (1996).

[9] L. Hartmann, B. Kraus, H.-J. Briegel, and W. Dür, Role of memory errors in quantum repeaters, Phys. Rev. A 75, 032310 (2007).

[10] L. Jiang, J. M. Taylor, K. Nemoto, W. J. Munro, R. Van Meter, and M. D. Lukin, Quantum repeater with encoding, Phys. Rev. A 79, 032325 (2009).

[11] Y. Li, S. D. Barrett, T. M. Stace, and S. C. Benjamin, Long range failure-tolerant entanglement distribution, New J. Phys. 15, 023012 (2013).

[12] A. Ahlbrecht, F. Richter, and R. F. Werner, How long can it take for a quantum channel to forget everything?, Int. J. Quantum. Inform. 10, 1250057 (2012).

[13] M. B. Plenio and S. Virmani, Spin Chains and Channels with Memory, Phys. Rev. Lett. 99, 120504 (2007).

[14] D. Kretschmann and R. F. Werner, Quantum channels with memory, Phys. Rev. A 72, 062323 (2005).

[15] N. Datta, J. M. Renes, R. Renner, and M. M. Wilde, Oneshot lossy quantum data compression, IEEE Trans. Inf. Theory 59, 8057 (2013).

[16] F. G. S. L. Brandao and J. Eisert, Correlated entanglement distillation and the structure of the set of undistillable states, J. Math. Phys. (N.Y.) 49, 042102 (2008).

[17] F. Buscemi and N. Datta, Distilling entanglement from arbitrary resources, J. Math. Phys. (N.Y.) 51, 102201 (2010).

[18] N. Datta and M.-H. Hsieh, One-shot entanglement-assisted quantum and classical communication, IEEE Trans. Inf. Theory 59, 1929 (2013).

[19] F. Leditzky and N. Datta, Second order asymptotics of mixed quantum source coding via universal codes, arXiv: 1407.6616.

[20] M. Wilde, A. Winter, and D. Yang, Strong converse for the classical capacity of entanglement-breaking and Hadamard channels via a sandwiched Rényi relative entropy, Commun. Math. Phys. 331, 593 (2014).
[21] F. Verstraete, J. J. Garcia-Ripoll, and J. I. Cirac, Matrix Product Density Operators: Simulation of Finite-Temperature and Dissipative Systems, Phys. Rev. Lett. 93, 207204 (2004).

[22] M. Zwolak and G. Vidal, Mixed-State Dynamics in OneDimensional Quantum Lattice Systems: A Time-Dependent Superoperator Renormalization Algorithm, Phys. Rev. Lett. 93, 207205 (2004).

[23] A. H. Werner, D. Jaschke, P. Silvi, T. Calarco, J. Eisert, and S. Montangero, A positive tensor network approach for simulating open quantum many-body systems, arXiv: 1412.5746 .

[24] J. Cui, J. I. Cirac, and M. C. Bañuls, Variational Matrix Product Operators for the Steady State of Dissipative Quantum Systems, Phys. Rev. Lett. 114, 220601 (2015).

[25] F. Verstraete, J. I. Cirac, J. I. Latorre, E. Rico, and M. M. Wolf, Renormalization-Group Transformations on Quantum States, Phys. Rev. Lett. 94, 140601 (2005).

[26] M. Fannes, B. Nachtergaele, and R. F. Werner, Finitely correlated states on quantum spin chains, Commun. Math. Phys. 144, 443 (1992).

[27] D. Perez-Garcia, F. Verstraete, M. M. Wolf, and J. I. Cirac, Matrix product state representations, Quantum Inf. Comput. 7, 401 (2007).

[28] U. Schollwöck, The density-matrix renormalization group in the age of matrix product states, Ann. Phys. (Amsterdam) 326, 96 (2011).

[29] J. Eisert, Entanglement in tensor network states, Model. Simul. 3, 520 (2013).

[30] R. Orus, A practical introduction to tensor networks: Matrix product states and projected entangled pair states, Ann. Phys. (Amsterdam) 349, 117 (2014).

[31] N. Schuch, Condensed matter applications of entanglement theory, arXiv:1306.5551.

[32] C. Schön, E. Solano, F. Verstraete, J. I. Cirac, and M. M. Wolf, Sequential Generation of Entangled Multiqubit States, Phys. Rev. Lett. 95, 110503 (2005).

[33] M. Kliesch, D. Gross, and J. Eisert, Matrix-Product Operators and States: NP-hardness and Undecidability, Phys. Rev. Lett. 113, 160503 (2014).

[34] C. H. Bennett, G. Brassard, S. Popescu, B. Schumacher, J. A. Smolin, and W. K. Wootters, Purification of Noisy Entanglement and Faithful Teleportation via Noisy Channels, Phys. Rev. Lett. 76, 722 (1996).

[35] R. Bhatia, Matrix analysis, Graduate Texts in Mathematics (Springer, New York, 1997).

[36] See Supplemental Material at http://link.aps.org/ supplemental/10.1103/PhysRevLett.116.020502, which includes Ref. [37], for outlined versions of the proofs, further mathematical concepts including an eigenvector perturbation theorem and the application of the 5-qubit protocol to correlated states using a physical toy model.

[37] N. Datta and T. Dorlas, The coding theorem for a class of quantum channels with long-term memory, J. Phys. A 40, 8147 (2007).

[38] S. Gudder, Quantum Markov chains, J. Math. Phys. (N.Y.) 49, 072105 (2008).

[39] O. Szehr and M. M. Wolf, Perturbation bounds for quantum Markov processes and their fixed points, J. Math. Phys. (N.Y.) 54, 032203 (2013). 